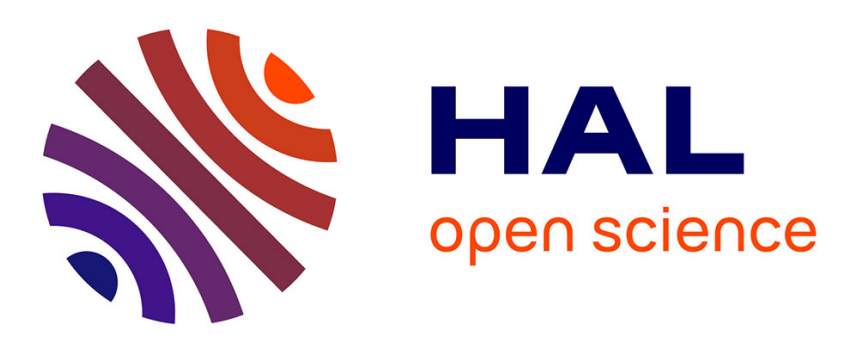

\title{
Integrating VANETs in the Internet Core with OSPF : the MPR-OSPF Approach
}

Emmanuel Baccelli, Thomas Heide Clausen, Philippe Jacquet, Dang-Quan

Nguyen

\section{- To cite this version:}

Emmanuel Baccelli, Thomas Heide Clausen, Philippe Jacquet, Dang-Quan Nguyen. Integrating VANETs in the Internet Core with OSPF : the MPR-OSPF Approach. 2007 7th International Conference on ITS Telecommunications, Jun 2007, Sophia Antipolis, France. pp.1-5, 10.1109/ITST.2007.4295864 . hal-02263429

\section{HAL Id: hal-02263429}

https://hal-polytechnique.archives-ouvertes.fr/hal-02263429

Submitted on 4 Aug 2019

HAL is a multi-disciplinary open access archive for the deposit and dissemination of scientific research documents, whether they are published or not. The documents may come from teaching and research institutions in France or abroad, or from public or private research centers.
L'archive ouverte pluridisciplinaire HAL, est destinée au dépôt et à la diffusion de documents scientifiques de niveau recherche, publiés ou non, émanant des établissements d'enseignement et de recherche français ou étrangers, des laboratoires publics ou privés. 


\title{
Integrating VANETs in the Internet Core with OSPF : the MPR-OSPF Approach
}

\author{
Emmanuel Baccelli*, Thomas Heide Clausen ${ }^{\dagger}$, Philippe Jacquet*, Dang-Quan Nguyen* \\ *INRIA, France. \{Emmanuel.Baccelli, Philippe.Jacquet, Dang-Quan.Nguyen\}@inria.fr \\ ${ }^{\dagger}$ Ecole Polytechnique, France. Thomas.Clausen@ polytechnique.fr
}

\begin{abstract}
Solutions for mobile ad hoc routing have matured over the last decade. Building atop these foundations, new challenges are set for MANETs, such as integration in the Internet core. On this topic, this paper designs and evaluates MPR-OSPF, an extension of the OSPF protocol enabling its operation on networks that may include both MANET nodes and usual fixed routers. Automatic integration of different types of vehicular ad hoc networks (VANETs) in the IP infrastructure is then possible using the classic OSPF framework. Techniques used therefore are derived from OLSR, the MANET routing protocol that is the most compatible with traditional IP environments.
\end{abstract}

\section{INTRODUCTION}

Mobile Ad hoc Networks have attracted considerable attention over the last decade, both in academic and industrial environments. MANETs were initially designed as networks isolated from the Internet (at most, MANETs would be connected via a gateway), and the main challenge was that of providing efficient routing inside MANETs, as the new characteristics implied by ad hoc mobility made traditional solutions inappropriate.

Two families of solutions have been developed in order to address this challenge. On one hand, the reactive routing protocols (such as AODV [3] and DSR [4]) and on the other hand the proactive routing protocols (such as OLSR [1]). These protocols have been the subject of intensive attention from the community in the recent years, and have been extensively experimented in various scenarii by means of simulations as well as actual deployments.

As MANET routing has matured, focus is now put on another challenge: integrating MANETs inside the Internet core, by means of designing a wireless extension for OSPF [2] enabling router ad hoc mobility. In order to guarantee full compatibility with legacy OSPF, a natural solution is to base this extension on MANETs' proactive link state approach, since OSPF is itself proactive and link state. In this paper we therefore design MPR-OSPF, a wireless extension for OSPFv3 based on OLSR, the MANET proactive protocol that has emerged as the simplest and most robust solution for mobile ad hoc routing.

\section{A. Paper Outline}

The paper is organized as follows. We first overview OLSR and the techniques it employs (it is however assumed that the reader is familiar with OSPF). Then, we describe the base of MPR-OSPF: the specification of a new OSPFv3 interface type tailored for MANETs. This new interface is plugging into OSPF several techniques derived from the IETF [16] developed MANET protocol OLSR, while retaining the basic OSPF framework. The performance of MPR-OSPF is then evaluated and compared to that of alternative approaches also under development, such as MDR [5] and OR [6].

MDR and OR propose OSPF extensions based on novel techniques, which deviate further from the generic OSPF framework. Qualitative arguments are then made about key differences in the design of MPROSPF compared with these other approaches. Finally, simulation results are given in order to make quantitative comparisons based on criteria including control traffic overhead, and delivery ratio in different node density and mobility scenarii.

We conclude from this qualitative and quantitative evaluation that MPR-OSPF is simple and efficient, as compared to the other proposed approaches. Moreover, we conclude that contrary to the other proposed approaches, MPR-OSPF ensures that its efficiency on MANETs is not at the price of altering traditional OSPF properties, and that in particular it preserves shortest path routing over synchronized links.

\section{THE OLSR PROTOCOL}

In this section we briefly outline OLSR, the proactive ad hoc routing solution standardized in the IETF [16]. The essential facts about the protocol that are interesting in the context of this paper are given in the following. For further details on OLSR, refer to [1].

As a proactive link-state routing protocol, OLSR (Optimized Link State Routing) employs periodic exchange of control messages in order to accomplish topology discovery and maintenance. This exchange results in a topology map being present in each node in the network, from which a routing table can be 


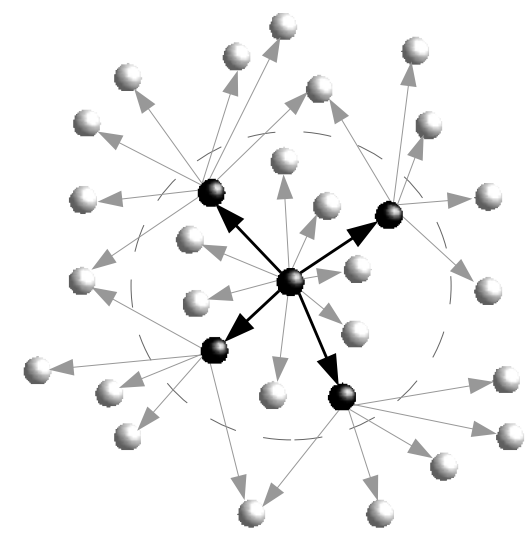

Fig. 1. Multipoint Relays of a node. A node (center) floods a message that is forwarded only by the neighbors it has selected as its MPRs (the black nodes). The range of the neighborhood of the node is depicted by the circle.

constructed.

Basically, OLSR employs two types of control messages: HELLO messages and TC (Topology Control) messages. HELLO messages have local scope and are exchanged periodically between neighbor nodes only, essentially tracking the status of links between neighbors. On the other hand, TC messages have larger scope and are emitted periodically to diffuse link-state information throughout the entire network. This operation of diffusing a message to the entire network - also called flooding - is optimized in OLSR with a mechanism called MPR flooding (MPR stands for Multi-Point Relay, see [10] for an analysis of this particular technique).

This optimization reduces drastically the cost of performing a flooding operation, through having each node select a minimal set of "relay nodes" (called MPRs), responsible for relaying flooded packets. As shown in Fig. 1, from the local point of view of a node flooding a packet - i.e. the center node in the figure - this corresponds to only the minimal number of neighbors (the black nodes) relaying the broadcast, instead of basically all the neighbors.

\section{OVERVIEW OF MPR-OSPF}

MPR-OSPF specifies an OSPFv3 interface type tailored for mobile ad hoc networks: the OSPF MANET interface. It is assumed that the reader is familiar with the OSPF protocol and related terminology [2]. The OSPF MANET interface makes use of flooding reduction, topology reduction and adjacency reduction mechanisms based on multi-point relaying (see previous section). The following outlines the design of this new interface type. For more details on MPR-OSPF, see [7].
Efficient Flooding with MPR. OSPF MANET interfaces use the MPR flooding technique, whereby only some OSPF MANET neighbors (those selected as MPR for flooding) are responsible for retransmitting a routing packet flooded over an OSPF MANET interface. This mechanism drastically reduces the number of (re)transmissions during flooding procedures, while still providing a natural high resilience in face of (i) transmission errors which are inherent to the use of wireless links, and (ii) obsolete two-hop neighbor information which is frequently caused by the mobility of routers [10] [11]

MPR Topology Reduction. OSPF MANET interfaces use a topology reduction mechanism called MPR topology reduction, whereby only necessary wireless links towards OSPF MANET neighbors (those concerned by MPR selection) are listed in LSAs. OSPF MANET routers periodically generate and flood Router-LSAs describing their selection of wireless links as point-to-point links to (current) OSPF MANET neighbors. This mechanism greatly reduces the size of LSAs originated by OSPF MANET routers, while still keeping OSPF's traditional properties: robust routing and optimal paths using synchronized adjacencies [12].

Multicast Transmissions of Protocol Packets. In order to reduce the overhead, multicast is used for most protocol packet transmissions over OSPF MANET interfaces, taking advantage of broadcast capabilities of the wireless medium. In particular, LSA acknowledgements are sent via multicast over these interfaces, and retransmissions over the same interfaces are considered as implicit acknowledgements. Intelligent jitter management delaying packets' (re)transmissions may also be used to increase the chance to bundle several packets in a single transmission, or to avoid superfluous retransmissions due to packet collisions.

MPR Adjacency Reduction. Furthermore, OSPF MANET routers may form adjacencies with a subset of its OSPF MANET neighbors (instead of all of them). However, no Designated Router or Backup Designated Router are elected on an OSPF MANET. Instead, routers must at least bring up adjacencies with their MPR and MPR selectors. Some select routers (called synch routers) must also bring up adjacencies with their other OSPF MANET neighbors. However other routers (most of them) do not have to bring up adjacencies with OSPF MANET neighbors other than their MPR and MPR selectors. This reduces the amount of control traffic needed for database synchronization, while ensuring that LSAs still describe synchronized adjacencies only. 


\section{Qualitative COMPARISON OF MPR-OSPF WITH ALTERNATIVE APPROACHES}

Aside from MPR-OSPF, other approaches are also being developed to specify a MANET interface for OSPF, including Overlapping Relays (OR [6]) and MANET Designated Routers (MDR [5]). This section compares these different approaches, from a qualitative point of view.

The MDR approach. This approach uses a biconnected dominating set (CDS) to reduce flooding overhead: only members of the CDS flood new LSAs back out the receiving interface. Adjacencies are formed only between members of the CDS and a subset of their neighbors. The CDS is constructed using the 2-hop neighbor information provided by a modified Hello protocol, which also allows differential Hellos that report only changes in neighbor states. A manually configurable option can further modify router-LSAs to provide partial topology information that still ensures reachability. For more information about MDR, see [5].

While both approaches are valid a priori, there is a main qualitative difference between MPR-OSPF and MDR concerning the properties of the routes that are provided. On one hand, MPR-OSPF (i) advertizes only synchronized adjacencies in LSAs and (ii) provides enough link information to derive shortest paths throughout the network, even if partial topology is used. On the other hand, the MDR approach (i) does not guarantee that links advertized in LSAs are all synchronized adjacencies, and (ii) does not guarantee that shortest paths can be derived, unless full topology information is used [12]. MDR's lacks in these domains may cause issues within the OSPF framework, as several mechanisms traditionally working along with OSPF are based on such guarantees regarding the quality of provided routes (e.g traffic engineering protocols). Moreover, MDR's use of unsynchronized adjacencies increases the probability of routing loops, which further degrades the quality of the routes provided by this solution.

The OR approach. This approach uses a modified MPR scheme to reduce flooding overhead: Overlapping Relays. A combination of scheduled retransmissions are set by neighbors which do not flood - these retransmissions are not fired only if the whole neighborhood has been covered and has acknowledged the flood before the scheduled time. OR uses a modified Hello protocol, which signals MPR selection and allows for incremental Hellos, that report only changes in the neighborhood. Finally, OR makes use of a mechanism called Smart Peering (SP), which reduces the number of adjacencies that are brought up, based on reachability information: an adjacency is not brought up with a neighbor which is aleady reachable in the routing table. For more information about OR, see [6].
As MPR-OSPF, the OR approach advertizes only synchronized adjacencies in LSAs. However, OR's adjacency reduction mechanism does not guarantee that enough link information is available to derive shortest paths throughout the network. Nevertheless, if their respective adjacency reduction mechanisms are not used, OR and MPR-OSPF approaches both provide a route quality that is similar to that of traditional OSPF. OR and MPR-OSPF approaches then differ only "technically", by (i) employing different flooding reduction mechanisms, and (ii) while MPR-OSPF uses a topology reduction mechanisms, OR does not. Therefore, it makes sense to compare OR and MPR-OSPF more precisely, and in order to do that, quantitative evaluation is needed. The next section provides such an evaluation.

\section{Simulation Results of the MPR-OSPF EXTENSION FOR MANETS}

This section compares, on a quantitative point of view, MPR-OSPF with OR, the alternative approach featuring similar route quality (see previous section). Simulation results using GTNetS [17] are presented, based on OSPFv3 Zebra code [15]. The parameters of the simulations reported in this paper are given in appendix.

The figures 2 through 9 compare the delivery ratio and the control traffic overhead in kbits per second generated by the OR protocol on one hand and the MPR-OSPF protocol on the other hand, without their respective adjacency reduction mechanisms for fair comparison (see previous section). Such evaluations are presented in scenarii featuring 20,30, 40 and finally 50 nodes in the network, with varying radio range.

These results show that while MPR-OSPF and OR provide similar delivery ratio in every simulated scenario, MPR-OSPF consistently outperforms OR in terms of overhead, by saving up to $60 \%$ overhead needed by OR to function.

\section{CONCLUSION}

In this document we have presented MPR-OSPF, an extension to OSPFv3 tailored for mobile ad hoc networks, accompanied by simulation results detailing the performance characteristics of the protocol. MPR-OSPF offers a basic plug-in of MANET matured techniques (based on MPR) in the OSPF framework, enabling OSPF to run efficiently over wireless MANET interfaces. The evaluations of the extension presented in this document show that MPR-OSPF is an excellent solution that outperforms alternative solutions, while remaining simple and robust, based on the marriage of OSPF with its natural counterpart in MANETs: OLSR. 


\section{REFERENCES}

[1] T. Clausen, P. Jacquet, Optimized Link State Routing Protocol Internet Engineering Task Force (IETF) Request For Comments, RFC 3626, October 2003.

[2] R. Coltun, D. Ferguson, J. Moy, OSPF for IPv6. Internet Engineering Task Force (IETF) Request For Comments, RFC 2740, December 1999.

[3] C. E. Perkins, E. M. Royer, S. R. Das, Ad Hoc On-Demand Distance Vector Routing. Internet Engineering Task Force (IETF) Request For Comments, RFC 3561, July 2003.

[4] D. Johnson, D. Maltz, Y. Hu, The Dynamic Source Routing Protocol for Mobile Ad Hoc Networks. Internet Engineering Task Force (IETF) Request For Comments, RFC 4728, February 2007.

[5] R. Ogier, P. Spagnolo, MANET Extension of OSPF using CDS Flooding. IETF Internet Draft draft-ogier-manet-ospf-extension, March 2007.

[6] M. Chandra et al. Extensions to OSPF to Support Mobile Ad Hoc Networking. IETF Internet Draft draft-chandra-ospf-manetext, January 2007.

[7] E. Baccelli, T. Clausen, P. Jacquet, D. Nguyen, OSPF MPR Extension for Ad Hoc Networks. Internet Engineering Task Force (IETF) Internet Draft draft-baccelli-ospf-mpr-ext, November 2006.

[8] A. Zinin, B. Friedman, A. Roy, L. Nguyen, D. Yeung, OSPF Link Local Signaling. Internet Engineering Task Force (IETF) Internet Draft draft-ietf-ospf-1ls, January 2007.

[9] E. Baccelli, T. Clausen, P. Jacquet, Ad-hoc and Internet Convergence: Adapting OSPF-style Database Exchanges for Ad-hoc Networks. Proceedings of the Conference on Performance Modelling and Evaluation of Heterogeneous Networks (HET-NETs), October 2004.

[10] A. Qayyum, L. Viennot, A. Laouiti, Multipoint Relaying: An Efficient Technique for Flooding in Mobile Wireless Networks. INRIA Research Report RR-3898, March 2000.

[11] C. Adjih, E. Baccelli, T. Clausen, P. Jacquet, On the Robustness and Stability of Connected Dominating Sets. INRIA Research Report RR-5609, June 2005.

[12] E. Baccelli, T. Clausen, P. Jacquet, Partial Topology in an MPR based Solution for Wireless OSPF on Mobile Ad Hoc Networks. INRIA Research Report RR-5619, July 2005.

[13] P. Jacquet, A. Laouiti, P. Minet, L. Viennot, Performance Evaluation of Multipoint Relaying in Mobile Ad Hoc Networks. Networking 2002 Proceedings, May 2002.

[14] J. Ahrenholz, E. Baccelli, T. Clausen, T. Henderson, P. Jacquet, P. Spagnolo, OSPFv2 Wireless Interface Type. Internet Engineering Task Force (IETF) Internet Draft draft-spagnolo-manet-ospfwireless-interface, May 2004.

[15] GNU Zebra, Free Routing Software distributed under GNU General Public Licence. http://www.zebra.org

[16] The Internet Engineering Task Force (IETF). http://www.ietf.org

[17] Georgia Tech Network Simulator (GTNetS). http://www.ece.gatech.edu/research/labs/MANIACS/GTNetS/

\section{PARAMETERS OF THE SimUlations}

The OR implementation used follows the specifications of [6], without the Smart Peering option. The MPR-OSPF implementation used follows the specifications of [7], without the adjacency reduction option.

The user traffic pattern used is CBR: 10 packets per second overall the network, each packet is 40 bytes long. Each node participates equally to the user traffic, by sending to each other node in the network the same fraction of the overall user traffic. The delivery ratio is then computed as the average of the delivery ratio for each node in the network. The other parameters used for the simulations that are reported in this paper are the following:

- Full IEEE $802.11 \mathrm{~b}$ : selected ranges 40m, 60m, ... $300 \mathrm{~m}$, with alpha : 0.5

- Field dimensions : 500m x 500m

- Number of nodes : 20, 30, 40, 50 nodes localized randomly on the field (uniform distribution)

- Mobility model: Random Waypoint, max speed $16 \mathrm{~m} / \mathrm{s}$, pause $1 \mathrm{~s}$

- HelloInterval : $2 \mathrm{~s}$

- Deadinterval : 6s

- RxmtInterval: $7 \mathrm{~s}$

- MinLSInterval : 5s

- MinLSArrival : $1 \mathrm{~s}$

- AckInterval : 500ms 


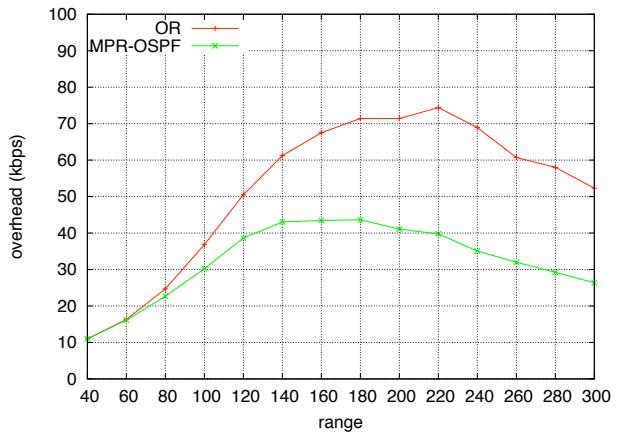

Fig. 2. Overhead with MPR-OSPF, compared with OR. 20 nodes.

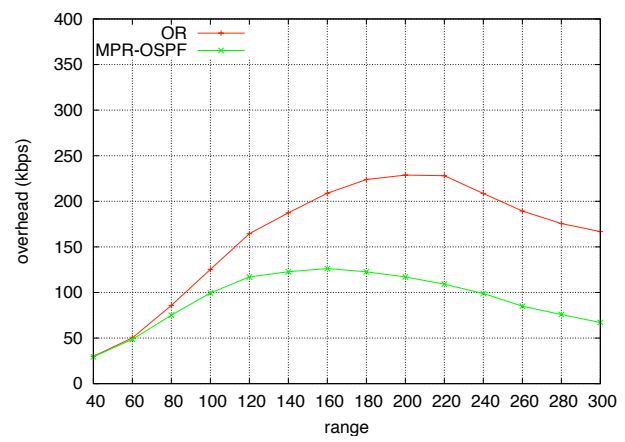

Fig. 3. Overhead with MPR-OSPF, compared with OR. 30 nodes.

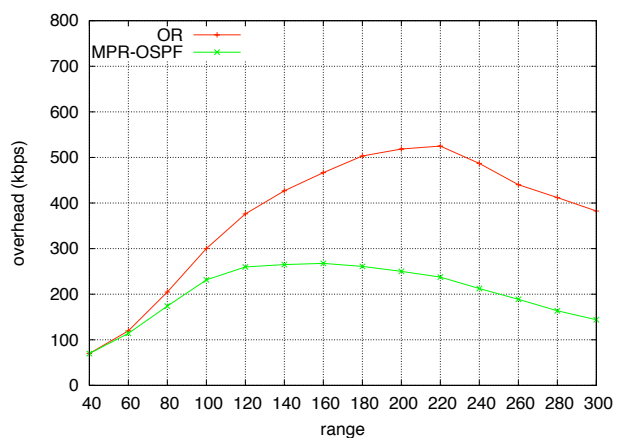

Fig. 4. Overhead with MPR-OSPF, compared with OR. 40 nodes.

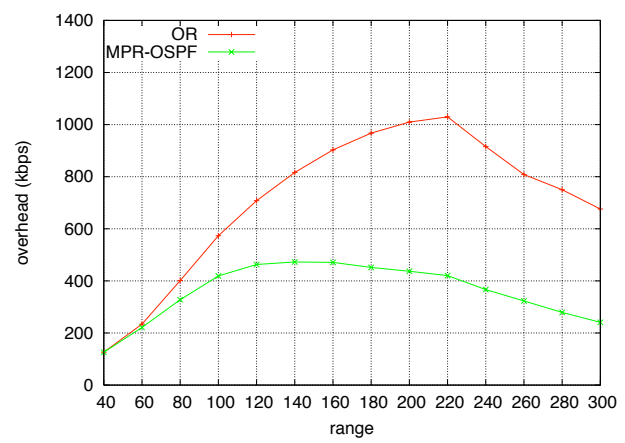

Fig. 5. Overhead with MPR-OSPF, compared with OR. 50 nodes.

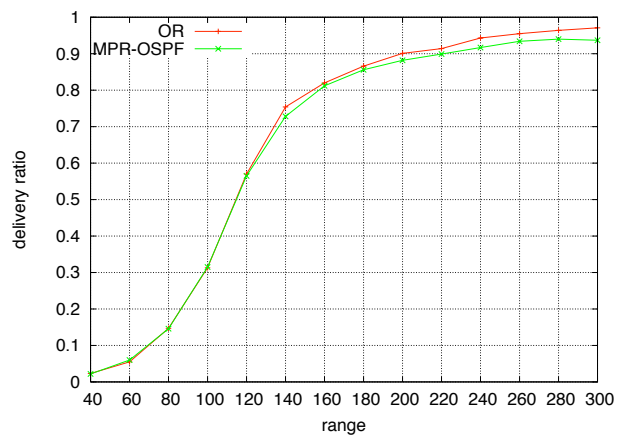

Fig. 6. Delivery ratio with MPR-OSPF, compared with OR. 20 nodes.

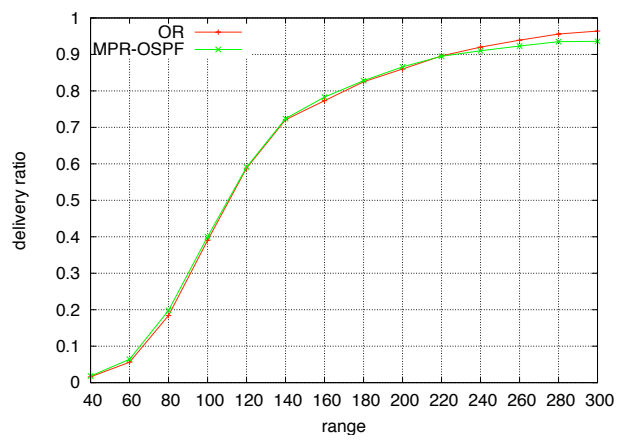

Fig. 7. Delivery ratio with MPR-OSPF, compared with OR. 30 nodes.

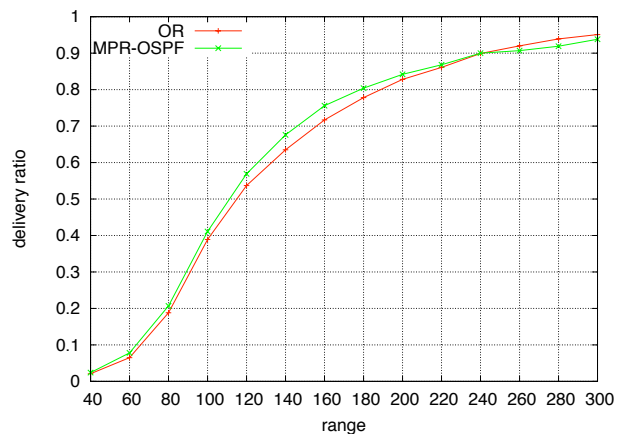

Fig. 8. Delivery ratio with MPR-OSPF, compared with OR. 40 nodes.

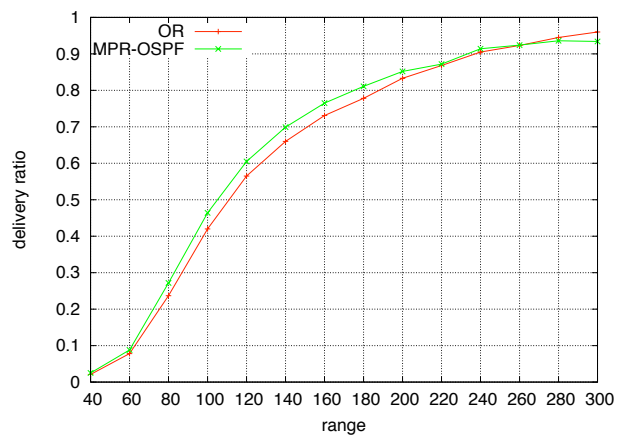

Fig. 9. Delivery ratio with MPR-OSPF, compared with OR. 50 nodes. 